\title{
The Intelligent Integration of Interactive Installation Art Based on Artificial Intelligence and Wireless Network Communication
}

\author{
Ze Gao ${ }^{1}$ and Lin $\operatorname{Lin}^{2}$ \\ ${ }^{1}$ Computational Media and Arts, The Hong Kong University of Science and Technology, HK, China \\ ${ }^{2}$ School of Creative Media, City University of Hong Kong, HK, China \\ Correspondence should be addressed to Ze Gao; zgaoap@connect.ust.hk
}

Received 2 August 2021; Revised 16 September 2021; Accepted 29 September 2021; Published 18 October 2021

Academic Editor: Zhihan Lv

Copyright (C) 2021 Ze Gao and Lin Lin. This is an open access article distributed under the Creative Commons Attribution License, which permits unrestricted use, distribution, and reproduction in any medium, provided the original work is properly cited.

\begin{abstract}
With the development of technology and the times, the development of new media technology and interactive installation art has slowly entered the vision of our audience. It is simply "silent art." The public no longer "retires" like the traditional one, but participates in it and swims with the artists in the world of art. This article is aimed at studying the application of artificial intelligence and wireless network communication to the application of interactive installation art. Through the optimization of various communication equipment and the continuous advancement of various algorithms, we can strengthen the communication and connection between our interactive installation art. This article proposes that with the addition of artificial intelligence and wireless network communication, the interaction between artists and audiences may be more fun, so that we can be more colorful in our lives. The experimental results in this article show that when performing wireless network communication, the communication delay rate of the intelligent algorithm with artificial intelligence is much lower than that of the one without it, which shows that they can better transmit information to the control end. When affected by the outside world, the bit error rate of wireless network communication will increase, however, the artificial intelligence algorithm is added to his impact range, and his bit error rate increase is obviously not so high. In the process of wireless network communication, the improved algorithm is definitely better than the nonimproved algorithm in terms of energy consumption, communication delay, and bit error rate. Through the enhancement of signals and the selection of materials for communication equipment, these are all in continuous progress, and in this respect, are in continuous exploration. Compared with other algorithms, the $\mathrm{ml}$ algorithm has improved positioning accuracy by about $70 \%, 65 \%$, and $30 \%$. Increasing the number of nodes in the transmission signal can greatly reduce the number of hops between nodes, correspondingly reducing the hop distance error, correspondingly reducing the distance estimation error, and improving the positioning accuracy. It can solve the technical barriers of interactive installation art faster.
\end{abstract}

\section{Introduction}

1.1. Background. With the development of the times, the advancement of science and technology, and the development of new media technology, interactive installation art has gradually entered the public eye and has become a new and energetic art form. In the changing times, the development of wireless network communication is becoming more and more mature. In the 1980s, the development of foreign sensors continued to grow. From a small aspect to a large range, our country started late, but afterward, we rapid development in this area. As well as people's understanding of the interactive art of installations, in the era of rapid development of artificial intelligence and wireless network communication technology, in order to enable artists and audiences to communicate and interact their emotions more intelligently, artificial intelligence, The new combination of wireless network communication technology is an inevitable product brought about by the development of the times, because it can satisfy the current exchange of emotions between our people and express the artworks created by artists [1]. The times are constantly progressing, and all 
industries are pursuing intelligent and convenient goals. Artificial intelligence represents the power of science and technology. We can know that at this time period, the power of science and technology is urging our progress.

1.2. Significance. Interactive installation art has been formed for nearly a century. It is produced in contemporary art in the context of the current era. Artists and audiences can experience and touch the works personally, and the interactivity is greatly improved. Technology and emotion interact to promote the relationship between art and the public [2]. Take the initiative to visit according to their own wishes rather than passively appreciate traditional works of art. Through this inspection, the public can actively experience the charm of characteristic art during the visit. With the addition of artificial intelligence and wireless network communication, interactive art installations can look more intelligent, and the effects displayed are also unique. Effectively add artificial intelligence and wireless network communication to interactive installation art, which not only allows us to better communicate with the masters of art but also makes it more convenient for artists to promote positive energy and allow audiences to truly communicate with art face-to-face. Give us more freedom and happiness.

1.3. Related Work. After reading a large number of related documents, the purpose of artificial intelligence is to make our lives more intelligent and to give machines a kind of human intelligence. The development of wireless network communication is also full of doubts and hardships, in order to be better. Feeding information back to users, and transmitting some important information in time, how many scientists have given their youth. The article uses the intelligent combination of artificial intelligence and wireless network communication in interactive installation art to better realize the emotional communication between art and the audience. In Fan and Zhang's research, they set up experiments to conduct neural network multisystem fusion, combined with big data and artificial intelligence, to verify the efficient operation of neural network in multisystem fusion. Use mathematical analysis and big data fitting methods to classify the collected data, collect experimental data, and then analyze. In the end, their work has improved the manufacturing industry by nearly $15 \%$. Although his research is very forward-looking, it is still difficult to apply in practice $[3,4]$. In their experiments, with the deepening of technology, the technical capabilities they possess have become more and more difficult, and the technology has become another challenge for them. In Zheng et al.'s research, they proposed a recurrent neural network algorithm based on long and short-term memory (LSTM) to recognize behavior patterns, thereby improving the accuracy of human activity behavior recognition. They used machine learning methods and the Internet of Things. But the learning methods they use are some relatively shallow learning methods. The experiment performs well in the laboratory environment, but it still needs to go a long way in practical applications [5]. In the research of Abed et al., they proposed an intelligent multimodal biometric verification model based on artificial intel- ligence method for identifying and verifying personal identity. The proposed model recognizes the unique patterns of each person's iris and finger veins to overcome many challenges such as identity fraud, poor image quality, noise, and instability of the surrounding environment. With the help of artificial intelligence, they successfully completed the experiment, but their experiment still has incomplete problems, and further research is needed [6]. In Fu et al.'s research, they proposed an improved hybrid game reinforcement learning algorithm, which takes the maximum controllable load participation as the objective function to achieve the optimal allocation of distributed energy at the microlevel. It reveals the law of group intelligent decision-making under the multitask of microgrid. There are still some limitations in their research [7]. In Xu et al.'s article, the orthogonal experiment method was used to obtain and analyze the acoustic emission and vibration acceleration signal data in the CNC machine tool processing under different tool wear levels and different cutting conditions. The wavelet envelope decomposition method is used to extract the best characteristic frequency bands of acoustic emission and vibration acceleration signals, and the characteristic parameters for identifying tool wear are determined. There are still certain technical barriers in research, which can be better applied to practice [8]. In Zhou et al.'s research, a text-driven fault diagnosis model is designed. Once the fault description text is entered, it can be classified and automatically judged which fault mode the fault described in the text belongs to. But they did not solve the shortcomings of text feature extraction methods that consider the text context and blindness to the topic of the corpus [9]. In Ji and Wakefield's research, they described how they used specific examples from the installations they exhibited to apply visualization, sound, and interaction design in their work with artificial ecosystems and living organisms. The article only describes how they use art installations and does not use relevant technology to support what they want to express [10]. Unlike them, the author will use wireless network communication and artificial intelligence to add to the interactive installation art at the same time, through this to increase the spread of interactive installation art.

1.4. Innovation. How to effectively combine artificial intelligence and wireless network communication, make art interaction more perfect in art communication, let more people understand art, and help them calmly deal with any difficulties and difficulties in life and work. Intelligence and machine learning technologies in the field of wireless communication have attracted much attention [11]. Artificial intelligence has achieved success in speech understanding, image recognition, and processing. The demand for wireless communication in a large number of application scenarios is growing and diversified. Artificial intelligence has become an important technology for these requirements. This document introduces some typical scenarios where artificial intelligence plays an important role in wireless communication, such as channel modeling, channel decoding, signal detection, and channel coding design, and discussed the relationship between information and artificial intelligence. 
Information theory. Then, wireless network communication artificial intelligence can be technically simpler and more comprehensive. Today, science and technology are becoming more and more advanced, solving many problems for mankind, and helping people save manpower and material resources. Some people say laziness creates the world. Because of this, there are many automated things in the world, including automatic washing machines, military drones, wireless networks, and satellite communication equipment in daily life. Wireless network communication overcomes many problems and waste of resources caused by traditional satellite communication systems in the wireless field. The equipment handles the problem by itself, which reduces the cost of data communication to a certain extent and saves resources. The article explains that if wireless networks and artificial intelligence are applied to interactive installation art, they must be applied to other devices. Make our lives more beautiful and convenient.

\section{Methods and Related Concepts}

2.1. Artificial Intelligence and Wireless Network Communication. Artificial intelligence, also known as mechanical intelligence, is a form of automatic control of equipment by humans, that is, automatic and regular control of the construction of equipment structure. The basic analysis of artificial intelligence mainly comes from the two aspects of "artificial," "intelligence," and "intelligence" (8220); "artificial" refers to the strategy of exploring mechanical production technology based on the goal of human behavior. Based on the production structure of mechanical programming control and production control, according to the needs of social development, combined with the requirements of procedural thinking and structural control [12]. With the gradual deepening of research and the development of social technology media, the practical form of artificial intelligence technology is gradually being promoted. The content of exploration includes philosophy, psychology, knowledge, etc., and applications also include fingerprint recognition, voice control, and other formats [13].

The English abbreviation of wireless sensor network is WSN. WSN technology is based on wireless communication and sensor technology and integrates built-in, distributed information processing technology, and other technologies to perform data collection, perception, control, and realtime monitoring in the area covered by the network. The wireless network communication network structure is shown in Figure 1 below [14].

Wired network is the carrier of data media. At the same time, the continuous accumulation of data has also become a prerequisite for the continuous level of intelligence in wireless networks. In the future, the wireless network will become an intelligent system with self-negotiation, self-optimization, and self-healing, and even a specific self-evolving system, as shown in Figure 2 below.

At present, immersive, interactive, and other multimedia art installations, as a new wave of art, have been integrated into all fields of modern life, leading the development trend of public art. Whether in parks, squares, or lively streets, those wonderful and interesting art installations not only bring unlimited creativity and interest to people, but even trigger deep thinking. Multimedia art installation is a comprehensive use of sound, light, shadow, etc., to perfect the art of science and technology and to explore and express the deep-seated needs between people and nature. Every art installation is an emotional exchange and dialogue between the artist and the audience. At present, artists all over the world are expressing their unique artistic concepts through various "installation art." Interactive installation art can not only show us the beauty of art but also attract the audience to the interactive connection, full of a great sense of participation. Through this two-way communication, the interest of participants can be increased. Compared with traditional communication methods, this method is more gentle and interesting. Now that science and technology are developing faster and faster, our pace of life is constantly accelerating. The development trend of artificial intelligence and wireless network communication is unstoppable. We have found that the application of artificial intelligence and wireless network communication in interactive installation art can make us interact better and truly understand the emotions and art expressed by the installation [15]. The following is the connection between artificial intelligence and wireless network as shown in Figure 3.

The theory of wireless network and sensor communication is a node problem that must be studied in the communication field. By using node connection points for communication, the data collected by wireless sensors can be quickly transmitted to the control end of the sensor network, and communication needs to be carried out according to the objective function [16]. The process of creating the objective function is as follows: in the wireless communication process of the sensor network, when the proportion of idle communication nodes exceeds $20 \%$, the calculation of the spatial position of the communication nodes can be calculated by the following formula:

$$
F= \begin{cases}\frac{\text { qer }}{q} & \text { qer }>0, \\ 0 & \text { qer } \leq 0 .\end{cases}
$$

In the above formula, qe can be used to describe the number of all idle communication nodes, and $q$ can be used to describe all nodes.

$$
\text { qer }=\mathrm{qe}-q \times 0.1
$$

In the process of wireless network communication, the use of all cluster head nodes has certain limitations. Assuming that each cluster head node helps nearby nodes to provide communication services at the same time, the 


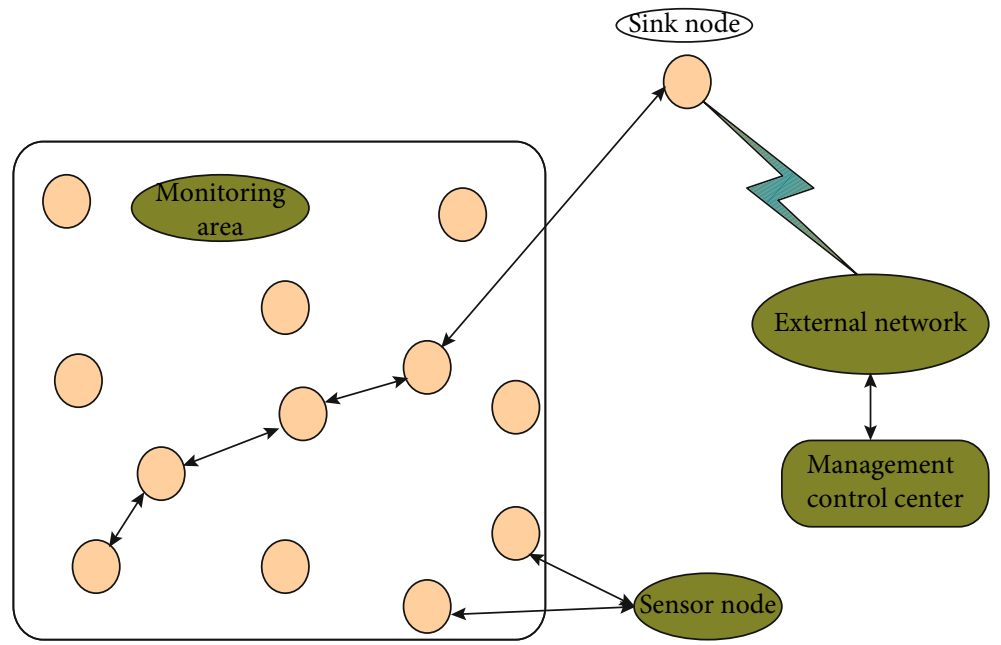

FIGURE 1: Network structure of wireless network communication.

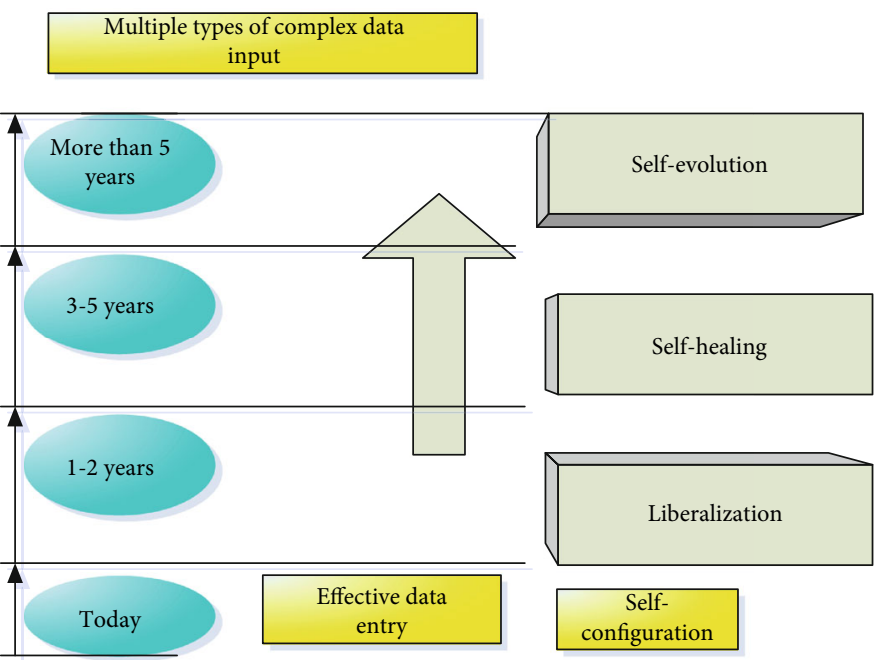

FIGURE 2: Intelligent improvement of wireless network.

following formula can explain:

$$
U=\frac{\sum_{K=1}^{\mathrm{BBC}} Q_{K}}{\mathrm{BBC}} \quad \begin{aligned}
& \mathrm{BBC}>0, \\
& \mathrm{BBC} \leq 0 .
\end{aligned}
$$

0

Group all the nodes in the wireless sensor network coverage area, and the number of nodes contained in each group is 3 , then these groups can use $(F 1, F 2, F 3),(F 4, F 5$ $, F 6), \cdots$ describe. If the remaining two nodes cannot participate in the grouping process, the above two nodes can be classified into one group [17]. If the remaining node cannot participate in the grouping process, the node is classified into a group [18]. The following formula can be used to calculate the ratio of nodes in different groups:

$$
F_{C K}\left[H_{11}=\frac{\sum_{K=1}^{3} L_{K} \cdot Z_{M K}}{\sum_{K=1}^{3} L_{K}}, A_{11}=\frac{\sum_{K=1}^{3} L_{K} \cdot A_{M K}}{\sum_{K=1}^{3} L_{K}}\right] .
$$

Through the method described above, we can obtain the ratios of these nodes. These ratios can be represented by the $F$ set. The average value of the node ratios of each group is $L_{1 M}$. Then after our calculations, we can get the wireless network communication optimal results.

$$
G\left(Z=\frac{\sum_{M=1}^{3} L_{K M} \cdot Z_{M K}}{\sum_{M=1}^{3} L_{K M}}, A=\frac{\sum_{M=1}^{3} L_{K M} \cdot Z_{M K}}{\sum_{M=1}^{3} L_{M}}\right) .
$$

After the above method, we can locate the optimal node for wireless network communication and combine the above optimal nodes to form a communication network and realize wireless sensor network communication [19].

Wireless communication systems are very different from wired network systems, but their channel state is not fixed, and any disturbance in transmission will affect the channel state, thereby affecting the transmission quality. As a signal transmission medium, the channel plays an important role in the MIMO communication system. The quality of 


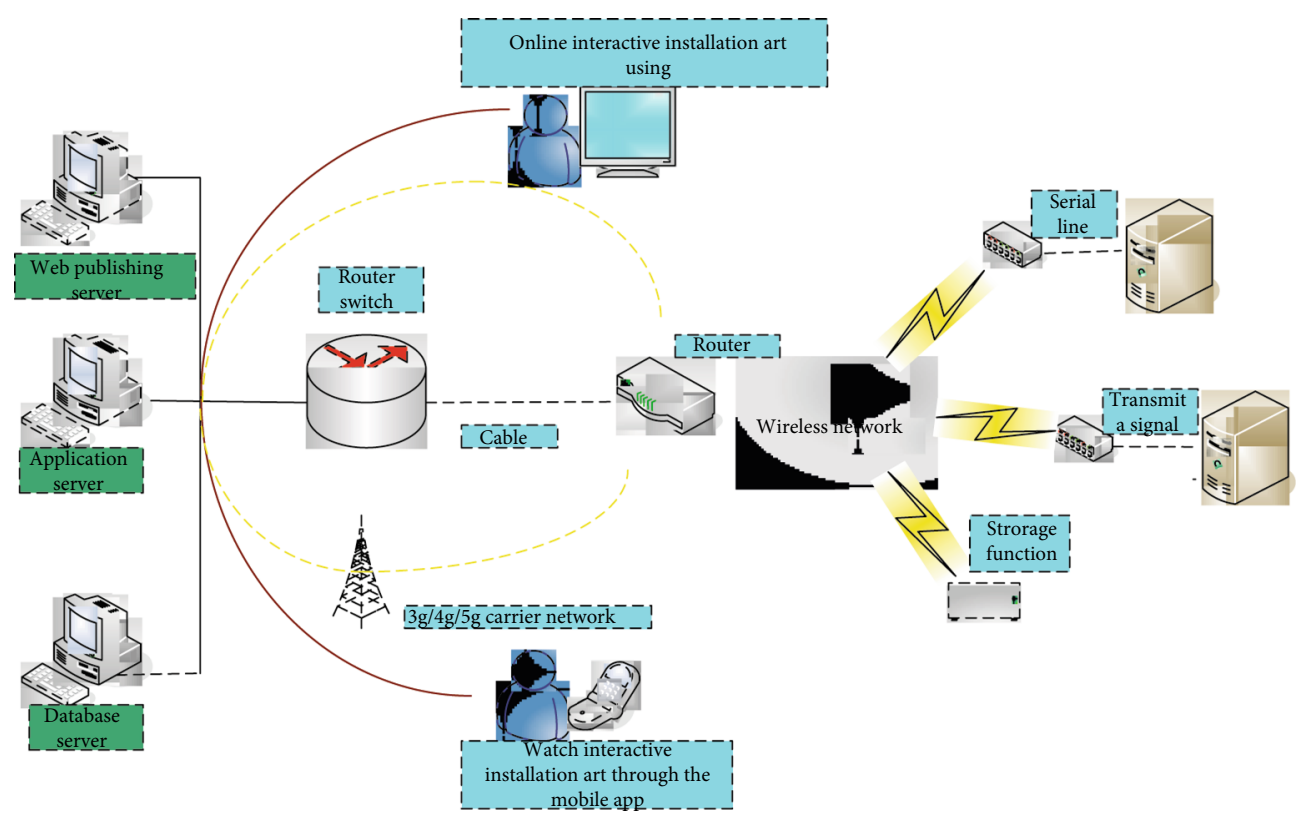

FIGURE 3: Communication between artificial intelligence and wireless network communication.

transmission and even the performance of the communication system are closely related to the channel state. Radio is usually used for mobile communication, which brings convenience to users and also brings difficulties to technical realization. Signals often appear "fading" in the channel and affect signal transmission quality. MIMO wireless communication systems also have channel fading characteristics, which mainly include the following two fading phenomena: large-scale fading and small-scale fading.

The causes of large-scale fading are path loss and shadow effect [20]. Path loss refers to the phenomenon that the energy of radio waves gradually attenuates with the increase of the propagation distance during the transmission process, resulting in the continuous weakening of the signal strength during the transmission process, which reduces the performance of the communication system and the transmission rate. This loss model can be used as follows express:

$$
j L(d)[d b]=20 \lg \left(\frac{4 \pi d_{0}}{\kappa}\right)+10 \mathrm{n} \lg \left(\frac{d}{d_{0}}\right) .
$$

In the above formula, where $d>d_{0}, d_{0}$ represents the reference distance, which is the wavelength of the transmitted signal, and $n$ is the path loss index (a constant determined by the specific environment), usually in the range of $2 \sim 6$ ((in free space) $n=2$ ). In the process of wireless signal propagation, we found that the signal will randomly react with objects and building obstacles, which will cause signal loss. In daily life, there are many reasons that cause the signal strength to become low, so usually use statistical models to represent these random attenuations. Among them, the measured data of the lognormal shadow model proves that the model can more accurately simulate the changes of the received power in the indoor and outdoor propagation environment [21]. In this model, it is assumed that the actual path loss exhibits a random change, and the change obeys a normal distribution. The probability density function of the received power decibel value is as follows:

$$
\operatorname{pr}\left(p L_{\mathrm{db}}\right)=\frac{1}{\sqrt{2 \pi \lambda_{\mathrm{PLDB}}}} \operatorname{EXP}\left(\frac{\left(P L_{d b}-\beta_{\mathrm{pldb}}\right)^{2}}{2 \lambda_{\mathrm{pldb}}^{2}}\right) .
$$

In equation (7), $\beta$ is the average path loss value at a given distance obtained in the model, $\lambda$ is the corresponding standard deviation, and the unit is $\mathrm{dB}$.

The small-scale fading reflects the dramatic changes in the energy and frequency of the signal in a short period of time or in a short distance, which is also called a fast fading phenomenon. Small-scale fading refers to fading in a short period of time. Specifically, when the mobile station moves a small distance, the received signal fluctuates rapidly in a short period of time. The small-scale fading is determined by many factors: multipath propagation, the speed of the mobile station, the speed of surrounding objects, and the signal transmission bandwidth. There are two main factors that cause fast fading. They are the Doppler effect caused by the rapid movement of some receiving devices, and the complex shapes of human living areas that cause multiple scattering and reflections during signal transmission. Distance propagation also has nonline-of-sight propagation, so that the "multipath effect" generated by the signal propagation through multiple different paths to the receiving device [22].

2.2. MIMO System Model. At present, MIMO technology has been widely used in the $4 \mathrm{G}$ mobile communication network LTE (Long Term Evolution) system. The most notable feature of MIMO technology is that it uses multiple antennas at the transmitting end and the receiving end, and multiple transmissions are realized at the receiving end. The signal 
diversity of the multitransmission channel path formed by the antenna performs reception and combination. In the MIMO system, due to the limitation of the physical size of the mobile station or the lack of scatterers at the base station, the spatial correlation between the antenna array elements will result. General spatial correlation is a function of the antenna's spatial structure, which includes several main parameters, such as angular spread, angle of arrival (DOA), transmit and receive scattering radius, and the distance between antennas. When the arrival angle of the antennas is the same, the larger the angle expansion, the stronger the scattering environment, and the smaller the correlation between the signals received by different antennas, and vice versa. In addition, when the angle extension is the same, the greater the arrival angle of the signal, the greater the correlation between the antennas, and vice versa. The MIMO system usually adopts the V-BLAST vertical layer-time code system model proposed by Bell Labs, as shown in Figure 4. This model converts the serially transmitted source data into multiple parallel data and sends them to each end of the transmitting antenna at the end of the transmission simultaneous transmission after differentiation. At the end of the download, the different symbols are separated by detection technology, which doubles the transmission speed of the system. It is a typical air separation multiplication system [23].

In traditional communication systems, the multipath effect is an unfavorable factor, but in the MIMO system, this has become an advantage. From this, we can infer that the MIMO system can combat the traditional multipath effect to a certain extent. The resulting fading, when we combine space-time coding and other technologies, we can effectively suppress the effect of channel fading to a certain extent to improve the performance of wireless network communication. The system model is shown in Figure 5 below.

Assuming that the system channel is a flat fading channel obeying the Rayleigh distribution, and any subtransmitting and receiving antennas have independent channel parameters, then, the MIMO signal sent at any time can be expressed in the form of a vector:

$$
x_{i}=\left[x_{i}^{1}, x_{i}^{2}, \cdots, x_{i}^{n}\right]^{i} .
$$

The corresponding received signal vector is

$$
y_{i}=\left[y_{i}^{1}, y_{i}^{2}, \cdots, y_{i}^{m}\right]^{i} .
$$

The obtained channel gain matrix $V$ is

$$
v_{i}=\begin{array}{cccc}
v_{11}^{i} & v_{12}^{i} & \cdots & v_{1 n}^{i}, \\
v_{21}^{i} & v_{22}^{i} & \cdots & v_{2 n}^{i}, \\
\vdots & \vdots & \ddots & \cdots \\
v_{m 1}^{i} & v_{m 2}^{i} & \cdots & v_{m n}^{i}
\end{array}
$$

Among them, $v_{i j}$ represents the total channel gain parameter from the $j$-th transmitting antenna to the $i$-th

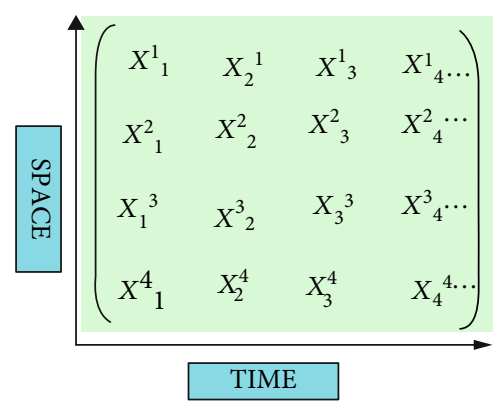

Figure 4: V-BLAST architecture.

receiving antenna, so the MIMO system model can be expressed as a simplified vector form:

$$
Y=V \cdot x+n .
$$

In the above formula, $n$ represents the interference term of the signal in the propagation channel. It is generally a random variable with a mean value of zero and a variance of $\alpha$. For our subsequent experiments and analysis, we generally transform the complex number domain model for real number domain models, and their general form is

$$
Y=\left[\frac{\mathrm{re}(y)}{\operatorname{im}(y)}\right]=\left[\frac{\mathrm{re}(h)-\operatorname{im}(h)}{\operatorname{im}(h)-\operatorname{re}(h)}\right]\left[\begin{array}{c}
\mathrm{re}(x) \\
\operatorname{im}(x)
\end{array}\right]+\left[\frac{\mathrm{re}(n)}{\operatorname{im}(n)}\right] \text {. }
$$

In the above formula, re and im both represent the real part and imaginary part of each selected matrix. Both $r e(x)$ and $\operatorname{im}(x)$ are interference items to the signal during the propagation process. In the subsequent research, we will work hard to optimize the propagation environment. Due to the multipath effect of wireless network communication, the signals received by the system will be superimposed, so the received signal on the $c$-th receiving antenna can be expressed by the following formula:

$$
Y_{c}=\sum_{i=1}^{N}\left(h_{c i} \cdot x_{i}\right)+n_{c} .
$$

This MIMO channel can be equivalently represented by $N$ mutually parallel subchannels, and the channel gain of each subchannel is equal to the singular value corresponding to the $V$ matrix. The channel capacity of any subchannel can be obtained by Shannon's formula:

$$
Z_{i}=B \log _{2}\left(1+\frac{P_{i}}{\sigma^{2}}\right)
$$

In the above formula, $\sigma^{2}$ represents the noise power, and $p$ represents the received power. When we are sending a signal, we do not know the channel state information, so when we allocate power, we mostly allocate it equally, assuming the total power is $R$, so there is, so the total capacity of the 


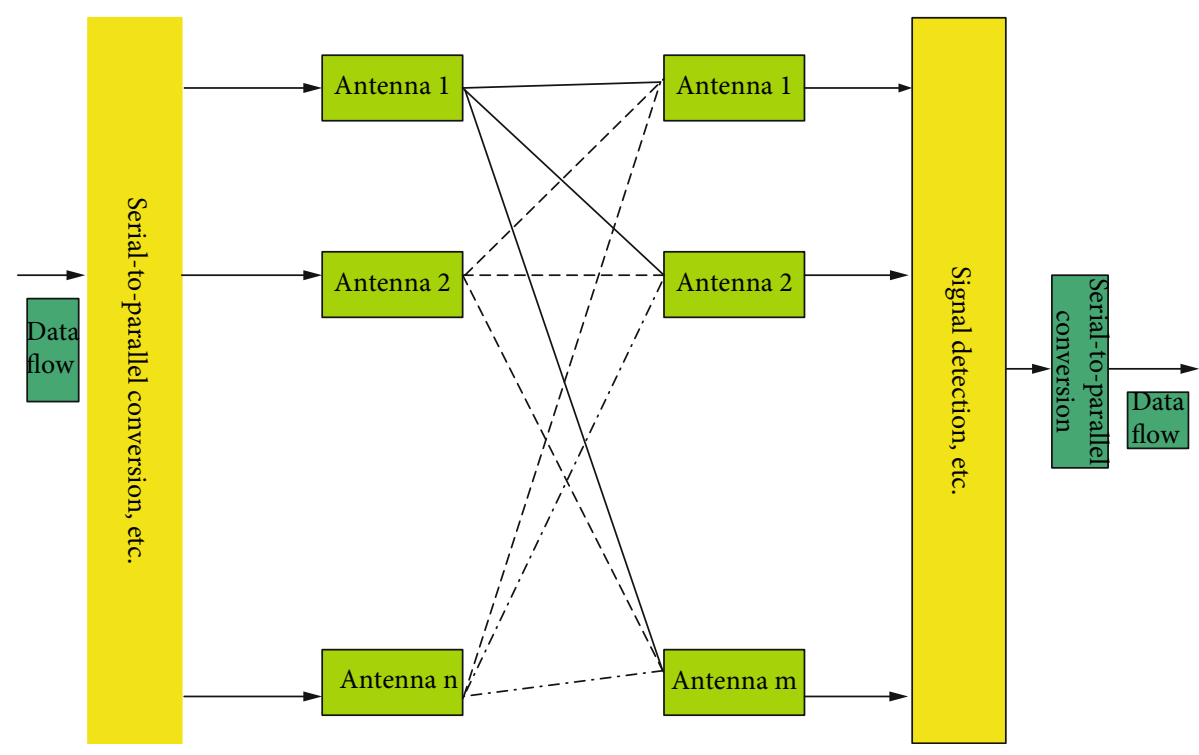

FIgURE 5: MIMO system structure diagram.

$R_{i}=\sqrt{\mu_{\mathrm{i}}} \cdot R / N$ channel is

$$
C=\sum_{i=1}^{N} c_{i}=b=\sum_{i=1}^{N}\left[\log _{2}\left(1+\sqrt{\mu_{i}} \cdot \frac{p}{\sigma^{2} n}\right)\right] .
$$

According to the special relationship between the feature matrix and the vector, we can get

$$
C=b \log _{2}\left[\operatorname{det}\left(I_{M}+\frac{\mathrm{SNT}}{N} \mathrm{VV}^{V}\right)\right] .
$$

In the experimental analysis, we generally set the bandwidth to be sufficient, but the channel state has a certain degree of randomness, so when we conduct the experiment, we generally choose the expected capacity as

$$
\bar{c}=e\left(\frac{c}{b}\right) E\left\{\mathrm{LOG}_{2}\left[\operatorname{det}\left(\mathrm{i}_{\mathrm{m}}+\frac{\mathrm{snt}}{n} \mathrm{vv}^{V}\right)\right]\right\} \text {. }
$$

2.3. Maximum Likelihood Monitoring Algorithm. The maximum likelihood detection algorithm is abbreviated as the ML algorithm. The maximum likelihood method explicitly uses a probability model, and its goal is to find a phylogenetic tree that can produce observation data with a higher probability. The maximum likelihood method is a representative of a class of phylogenetic tree reconstruction methods based entirely on statistics. The core idea of the algorithm is to maximize the conditional probability that the transmitted signal is $x$ when the received signal is $y$, that is, because the system model is

$$
Y=K X+n \Rightarrow n=y-k x \text {. }
$$

It can generally solve classification problems (discrete case) and regression problems (continuous case). It is a tree structure for processing samples, which is composed of nodes and directed edges. Because the idea is relatively simple, its interpretability is relatively strong, and the classification speed is relatively fast, but the overfitting phenomenon is also very serious, so there will be subsequent pruning operations. In the formula, $n$ is a random variable with a mean value of zero and a variance of $\sigma^{2}$, which is $n \sim N(0$, $\sigma^{2}$ ). When the parameter $k$ is known, then the conditional distribution conforms to the following distribution:

$$
p(y \mid x)=\frac{1}{2 \pi \eta} e^{|y-k x|^{2} / 2 \sigma^{2}} .
$$

The maximized probability $p(y \bowtie x)$ is equivalent to min $\|y-k x\|^{2}$, then, the $\mathrm{ml}$ algorithm can be expressed by the following formula:

$$
\min \|y-k x\|^{2}
$$

The $\mathrm{Ml}$ algorithm is a monitoring system with the best performance. It can minimize the overall error rate seen. Its complexity is directly related to the size of the space where the signal is received. As the number of antennas to be transmitted increases, the number of antennas also increases. With the continuous increase, the $\mathrm{ml}$ algorithm also has an exponentially increasing complexity, so it cannot be applied to actual communication systems, and is used as a performance comparison standard for other optimal algorithms.

\section{Comparative Experiment}

3.1. Experimental Environment and Parameter Settings. In order to verify the effectiveness and feasibility of the algorithm in the article, the article simulates the algorithm on the $2.50 \mathrm{GHz}$ Intel (R) Corei7 CPU and $16 \mathrm{~GB}$ RAM, Matlab2010a simulation platform, and compares the 
simulation results of the algorithm in this article and the RSSI trilateral test. The distance positioning algorithm and the RSSI weighted centroid positioning algorithm are compared [24]. The simulation environment is that the nodes are uniformly and randomly distributed in a rectangular area of $100 \mathrm{~m} \times 100 \mathrm{~m}$, and the experimental parameters used are shown in Table 1 below. The experimental data is the data measured by the machine in our laboratory for related experiments. In order to make the data better for comparison, we make pictures for relevant analysis.

3.2. The Relationship between the Number of Nodes and the Positioning Accuracy. The experimental method uses field detection methods to perform certain calculations on these algorithms and then draws graphs based on the data measured by the experiment, which can more intuitively observe the results of our research. The relationship between the number of nodes and the positioning accuracy is to simulate the positioning accuracy of the improved algorithm under the premise that the parameters remain unchanged and compare the traditional RSSI ranging trilateral positioning algorithm and the weighted centroid positioning algorithm [25]. The number of anchor nodes changes from 5 to 50 , and the relationship between the positioning accuracy and the number of anchor nodes is shown in Figure 6.

The overall placement accuracy of the ML algorithm is better than the other two algorithms. With the increase in the number of anchor nodes, the position accuracy of the three methods has also improved. When the number of anchor nodes is small, the position accuracy of this algorithm is higher than the other two algorithms, and the position accuracy is improved by about $50 \%$ compared with the traditional three-part RSSI. Compared with the detection algorithm, the position coverage changes with the number of nodes are given. When the number of nodes is 5 , the traditional trilateral positioning algorithm can no longer locate the nodes, and the positioning coverage is basically 0 [26]. As the number of nodes increases, the error of the $\mathrm{ml}$ algorithm in Figure 6 gradually approaches 0. In Figure 6(b), we can see that with the increase in the number of anchor nodes, the improved algorithm can achieve faster high coverage rate, when the anchor node density is $30 \%$, and the coverage rate is close to $100 \%$.

3.3. Algorithm Calculation Time Comparison. As shown in Table 2, when the communication radius is $30 \mathrm{~m}$, the number of nodes is $100,150,200,250$, and 300 , the number of nodes is $10 \%$ of the number of nodes, and the simulation time of the three algorithms increases with the number of nodes. In the table, we can clearly see that with the increase of the number of nodes, the time of wcp is also slowly from 0.55 to $2.96 \mathrm{~s}$, the difference is that both $\mathrm{rssl}$ and $\mathrm{ml}$ reached 130-145 s. Increase can be seen from the data results that the computational complexity of the weighted centroid positioning algorithm is much lower than the other two algorithms. Although the positioning algorithm in this paper uses the particle swarm optimization algorithm, it does not increase the computational complexity of the algorithm. The computational complexity of the overall algorithm is
TABLE 1: List of experimental parameters.

\begin{tabular}{lcc}
\hline Parameter & Meaning & Parameter value \\
\hline$R$ & Communication radius & 35 \\
Time & Simulation times & 200 \\
$T$ & Signal acquisition times & 200 \\
$N$ & Number of particles & 35 \\
$k \max$ & Population iterations & 35 \\
$c 1$ & Learning factor & 2 \\
$c 2$ & Learning factor & 2 \\
$w$ & Inertia weight & 0.85 \\
$n$ & Path attenuation index & 2.5 \\
$\sigma$ & Gaussian noise standard deviation & 2 \\
$d 0 / m$ & RSSI reference distance & 2 \\
\hline
\end{tabular}

slightly lower than the traditional trilateral positioning algorithm. RSSI and wcp measure the distance between the signal point and the receiving point by the strength of the received signal and then perform positioning calculations based on the corresponding data. For example, the positioning engine of the wireless sensor ZigBee network CC2431 chip uses this technology and algorithm. The receiver measurement circuit obtains an indication of the average signal strength of the receiver input. This measurement usually does not include antenna gain or transmission system loss.

As shown in Figure 7, it can be seen from Figure 7(a) that the average error of various algorithms is decreasing as the number of our anchor nodes increases. Compared with other algorithms, the $\mathrm{ml}$ algorithm has improved positioning accuracy by about $70 \%, 65 \%$, and $30 \%$. Increasing the number of nodes in the transmission signal can greatly reduce the number of hops between nodes, correspondingly reducing the hop distance error, correspondingly reducing the distance estimation error, and improving the positioning accuracy [27]. In Figure 7(b), we change the number of nodes from 100 to 300 , the communication radius is $30 \mathrm{~m}$, and the number of anchor nodes is always $10 \%$ of the number of nodes. As the number of nodes increases, the density of nodes in the network also increases, and the calculation of the hop distance becomes more accurate, so the positioning error tends to decrease.

\section{Discussion of Experimental Results}

4.1. Comparative Discussion. In the wireless sensor network communication process, energy consumption is an important indicator to measure the communication algorithm. This energy consumption includes two parts, namely, the energy consumed during the data transmission process of the communication node and the energy consumed during the communication data gain process. The traditional algorithm and the algorithm in this paper are used for wireless sensor network communication, and the comparison of energy consumption after artificial intelligence algorithm is added to the wireless network can be described with a graph 8 . 


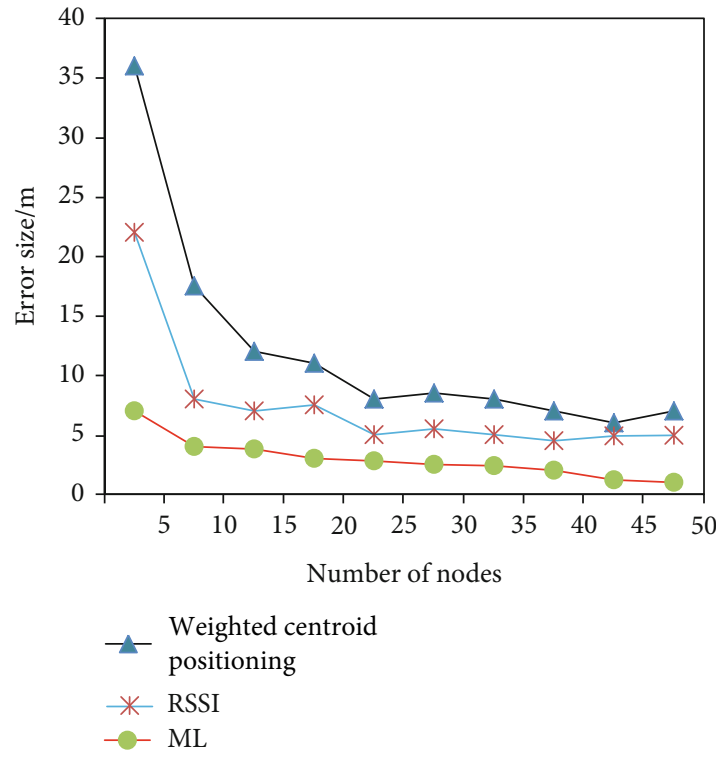

(a)

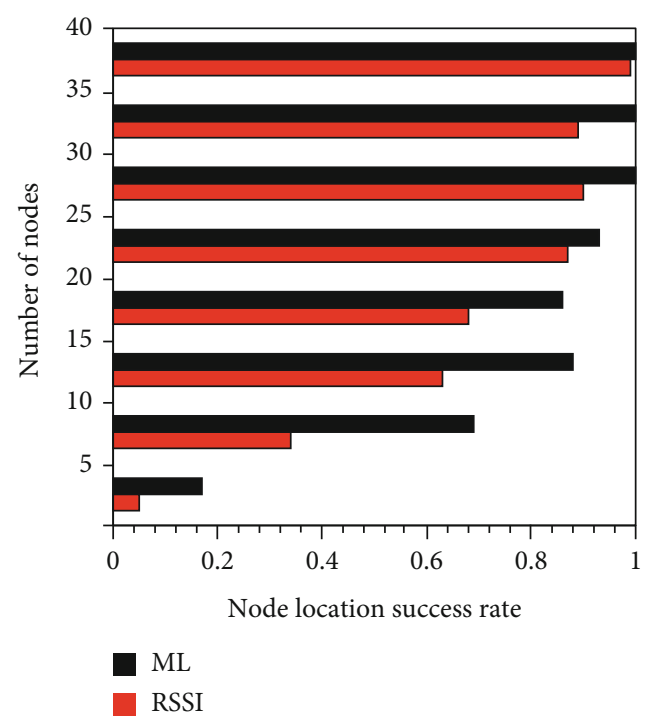

(b)

Figure 6: (a) The relationship between the number of nodes and the positioning accuracy. (b) The relationship between the number of nodes and the positioning coverage.

TABLe 2: Algorithm time comparison.

\begin{tabular}{lccccc}
\hline Number of nodes & 100 & 150 & 200 & 250 & 300 \\
\hline WCP & $0.55 \mathrm{~S}$ & $0.95 \mathrm{~S}$ & $1.56 \mathrm{~S}$ & $2.55 \mathrm{~S}$ & $2.96 \mathrm{~S}$ \\
RSSI & $69.5 \mathrm{~S}$ & $100.22 \mathrm{~S}$ & $121 \mathrm{~S}$ & $130 \mathrm{~S}$ & $145.2 \mathrm{~S}$ \\
$\mathrm{ML}$ & $50.12 \mathrm{~S}$ & $80.23 \mathrm{~S}$ & $100.23 \mathrm{~S}$ & $110.23 \mathrm{~S}$ & $130.56 \mathrm{~S}$ \\
\hline
\end{tabular}

According to the trend shown in Figure 8(a), we can know that when performing wireless network communication, the communication delay rate of the intelligent algorithm with artificial intelligence is much lower than that of the nonjoin, which shows that they can better integrate the information is transmitted to the control terminal. In Figure 8 (b), the improved algorithm for communication delay is lower than $\mathrm{ml}$ on the same number of nodes, ranging from 0.03 to 0.09 . These are subtle differences, but this reduces it to $0.02-0.069$. It can be concluded that the bit error rate of wireless network communication will increase when it is affected by the outside world, but the impact of artificial intelligence algorithm is not so large, and its bit error rate increase is obviously not that high. In the process of wireless network communication, the improved algorithm is definitely better than the unimproved algorithm in terms of energy consumption, communication delay, and bit error rate [28].

4.2. Interactive Installation Art under Intelligent Integration. After a series of algorithm experiments, according to the optimal combination algorithm, the expression of interactive installation art is becoming richer and richer with the rapid development of science and technology. Nowadays, mobile phones, computers, tablets, TVs Wait, all can be used.
Communicating with artists is not just verbal communication, but can be sublimated to emotional communication. Now through these intelligent algorithms, these devices can transmit faster and more clearly, no matter where you are, the network is ubiquitous. Even if we did not watch it at the beginning, we can also watch it through playback. Correspondingly, this can make the artwork not only bloom for a moment, and they can make the artwork exist for a longer time. With the application of technology, interactive devices will be more suitable for our lives and work. The current way of transmission is roughly as shown in Figure 9.

The time of the wireless sensor network location can be calculated. Through this research, it can be concluded that the use of artificial intelligence optimization algorithms will increase the complexity of calculation time and affect the energy of nodes. The focus of the next step is to find more suitable methods to reduce individual feasibility space, improve position accuracy and algorithm convergence, and meet the requirements of high-precision detection. Artists can create through video and audio. Interactive installation art can cover many fields. For example, the Mendelssohn Memorial Hall, a famous musician and conductor in Leipzig, Germany, has a new type of interactive media device called the Mendelssohn effector. This is a virtual orchestra. Visitors can use this device to simulate famous conductors like Mendelssohn. Its technical means is that visitors hold the baton signal, capture images through the $3 \mathrm{D}$ motion detection camera, and control the music speakers in front. People can control the speed and rhythm of the orchestra in realtime like a conductor and change the real-time performance of orchestral music by waving the baton. Concert hall and so on. This kind of interactive device embodies that its application of cross-border devices can allow originally unrelated elements to penetrate and merge with each other, giving 


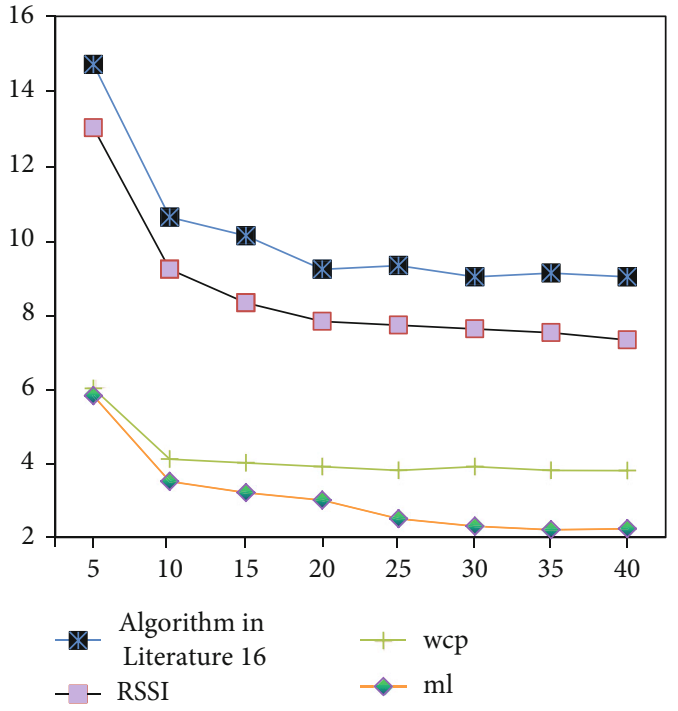

(a)

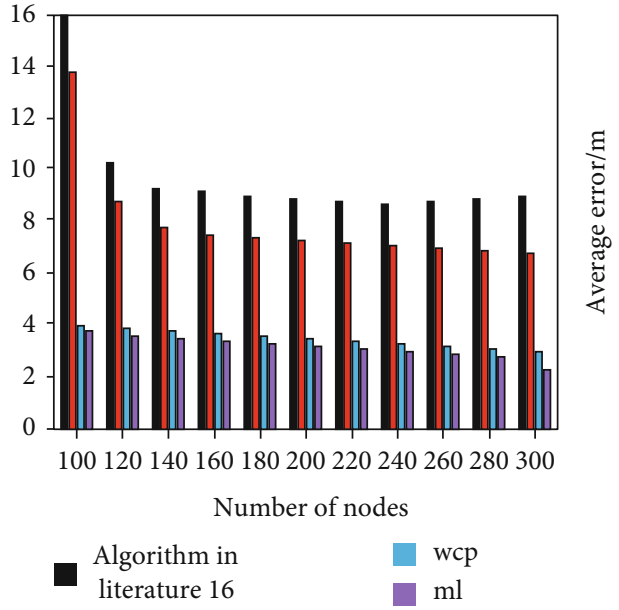

RSSI

(b)

FIGURE 7: (a) Variation of average positioning error with the number of anchor nodes. (b) Variation of average positioning error with communication radius.

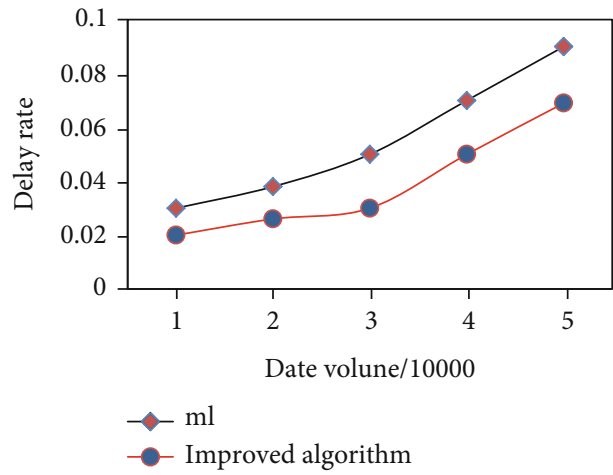

(a)

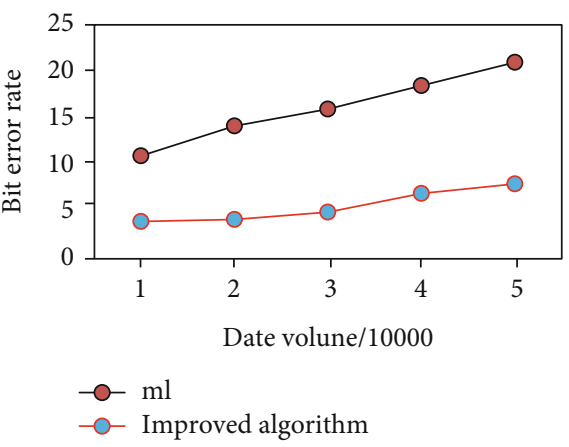

(b)

Figure 8: (a) Delay rate comparison. (b) Bit error rate comparison.

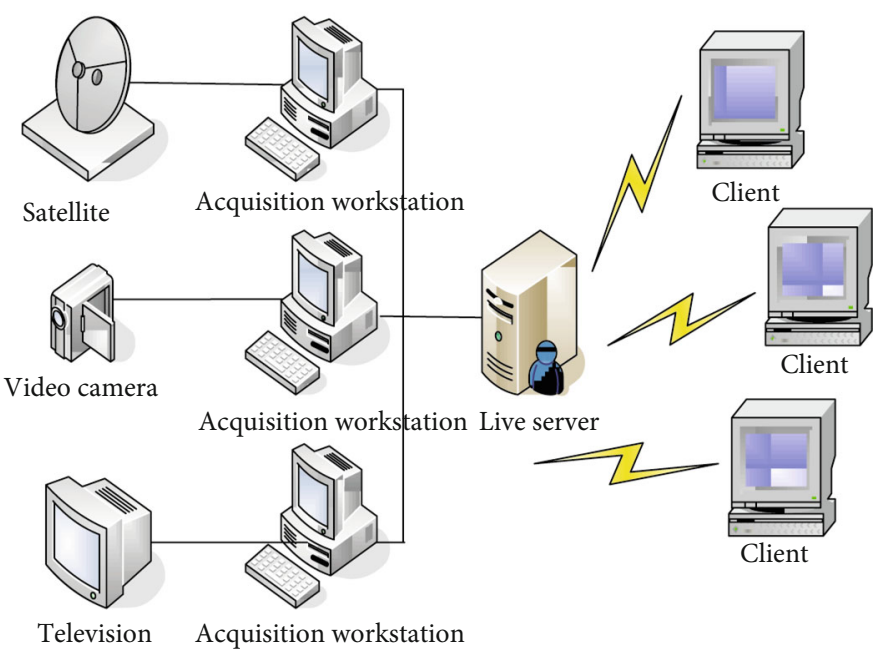

FIGURE 9: Interactive installation art dissemination. 
people an immersive sense of freshness, and it is a combination of technology and art [29].

\section{Conclusions}

In wireless network applications, location information can not only tell users where things are happening but can also be used to track targets and predict trajectories. The sensors are limited and develop randomly in the monitoring environment, resulting in uneven node density. Therefore, it is very important to design an efficient node detection algorithm. This article examines the anchor node density, placement accuracy, coverage, and complexity of the wireless sensor network algorithm. The technical public cannot respond to the news. Objective attitude: the emergence of new media has left traditional media without a foothold. This requires television to use technology to find a way to survive in the tide of this era and to find the main body suitable for the development and dissemination of traditional news. 8220 "VR + news" has become a new form of news, there is no other access method, at the horizontal data level, it mainly plays a role of data aggregation, and then according to SDM, the data of this process can be used to process data in real time. It is necessary to ensure that these data formats are consistent and can be accessed through the integrated client API. The ability to deepen data through the various business needs of hospital financial management is conducive to the effective promotion of financial management, scientific and effective decision-making, and the integration and innovation of technology and art, which promotes the development of new means of interactive devices in the hospital. In the current era, technological innovation is constantly advancing the trend of design art towards differentiation and diversified media. In the future life of science and technology, our technology will only become more developed and smarter. The emotional energy expressed by art can stimulate our potential. In the future, our lives will be more and more. The more intelligent. In this development process, with the help of technological advancement, interactive installation art has left many memorable and admirable extraordinary works of art: in the long run, they are priceless. The unique functions of the new interactive media equipment fully meet the visual needs required for the operation of contemporary digital art exhibitions and meet the sensory needs of the public at different levels when participating in the interactive experience. Under the development of artificial intelligence, our living conditions are constantly improving. The development of wireless networks has prompted their integration. In the research of this article, various wireless network algorithms have been compared and tested with the purpose of exploring the most energy-efficient, powerful equipment. The advancement of science and technology will also drive the in-depth development of new media interactive devices in various fields and multiple dimensions. There are still many deficiencies in our article. When we experiment, we will find that we have excluded some of these factors that affect the results of the experiment, but because we cannot eliminate them, we can only ignore them, correspondingly. The experimental results obtained are flawed, so it needs to be considered more comprehensively in subsequent research, and the level of knowledge should also be broad. The fusion of artificial intelligence and wireless network communication applications will undoubtedly bring convenience to our human lives in the future, but while bringing convenience to us, it will also bring us a certain amount of laziness. As working people, our lives are advancing, and it cannot be just satisfied with the present and stagnating. The times need continuous progress; otherwise, they will be overwhelmed by the trends of the times.

\section{Data Availability}

The data that support the findings of this study are available from the corresponding author upon reasonable request.

\section{Conflicts of Interest}

The authors declared no potential conflicts of interest with respect to the research, authorship, and/or publication of this article.

\section{References}

[1] Y. Sun, H. Song, A. J. Jara, and R. Bie, "Internet of things and big data analytics for smart and connected communities," IEEE Access, vol. 4, pp. 766-773, 2016.

[2] Z. Cai and Z. He, "Trading private range counting over big IoT data," in The 39th IEEE International Conference on Distributed Computing Systems, ICDCS, 2019.

[3] L. Fan and L. Zhang, "Multi-system fusion based on deep neural network and cloud edge computing and its application in intelligent manufacturing," Neural Computing and Applications, vol. 2, pp. 1-10, 2021.

[4] S. Yang, B. Deng, J. Wang et al., "Scalable digital neuromorphic architecture for large-scale biophysically meaningful neural network with multi-compartment neurons," IEEE transactions on neural networks and learning systems, vol. 31, no. 1, pp. 148-162, 2020.

[5] B. Zheng, D. Yun, and Y. Liang, "Research on behavior recognition based on feature fusion of automatic coder and recurrent neural network," Journal of Intelligent Fuzzy Systems, vol. 39, no. 6, pp. 8927-8935, 2020.

[6] E. A. Abed, R. J. Mohammed, and T. Shihab, "Intelligent multimodal identification system based on local feature fusion between iris and finger vein," Indonesian Journal of Electrical Engineering and Computer Science, vol. 21, no. 1, pp. 224232, 2021.

[7] X. Fu, H. Wang, Z. Wang, Z. Shi, W. Yang, and P. Ma, "Research on micro-grid group intelligent decision mechanism under the mode of block-chain and multi-agent fusion," Energies, vol. 12, no. 21, pp. 4196-4200, 2019.

[8] Y. Xu, L. Chen, Z. Yuan, T. Xie, and CASIA, "Intelligent recognition of tool wear conditions based on the information fusion," Zhendong yu Chongji/Journal of Vibration and Shock, vol. 36, no. 21, pp. 257-264, 2017.

[9] S. Zhou, B. Chen, Y. Zhang, H. X. Liu, Y. Xiao, and X. Pan, "A feature extraction method based on feature fusion and its application in the text-driven failure diagnosis field," 
International Journal of Interactive Multimedia and Artificial Intelligence, vol. 6, no. 4, pp. 121-123, 2020.

[10] H. Ji and G. Wakefield, "Endogenous biologically inspired art of complex systems," Computer Graphics \& Applications IEEE, vol. 36, no. 1, pp. 16-21, 2016.

[11] Z. Xu and Z. Cai, "Privacy-preserved data sharing towards multiple parties in industrial IoTs," IEEE Journal on Selected Areas in Communications (JSAC), vol. 38, no. 5, pp. 968-979, 2020.

[12] R. Li, X. Li, and Y. Zhang, "Fault diagnosis of multiinformation fusion in train intelligent control system based on fuzzy neutral network and evidential theory," International Journal of Control and Automation, vol. 9, no. 3, pp. 375-382, 2016.

[13] Y. Fu, S. Wang, C. X. Wang, X. Hong, and S. McLaughlin, "Artificial intelligence to manage network traffic of $5 \mathrm{G}$ wireless networks," IEEE Network, vol. 32, no. 6, pp. 58-64, 2018.

[14] Y. Jin, H. Gao, T. Hu, and X. Li, "Special issue on AI-driven smart networking and communication for personal internet of things, part I," International Journal of Wireless Information Networks, vol. 26, no. 3, pp. 131-132, 2019.

[15] S. Seo, D. Ko, and J. Chung, "Combined time bound optimization of control, communication, and data processing for FSObased 6G UAV aerial networks," ETRI Journal, vol. 42, no. 5, pp. 700-711, 2020.

[16] K. Hwang, M. Chen, H. Gharavi, and V. C. Leung, "Artificial intelligence for cognitive wireless communications," Wireless Communications, IEEE, vol. 26, no. 3, pp. 10-11, 2019.

[17] K. Gai, K. Xu, Z. Lu, M. Qiu, and L. Zhu, "Fusion of cognitive wireless networks and edge computing," IEEE Wireless Communications, vol. 26, no. 3, pp. 69-75, 2019.

[18] S. K. Mohanty and S. K. Udgata, "SATPAS: SINR-based adaptive transmission power assignment with scheduling in wireless sensor network," Engineering Applications of Artificial Intelligence, vol. 103, no. 11, p. 104313, 2021.

[19] A. Zappone, M. di Renzo, M. Debbah, T. T. Lam, and X. Qian, "Model-aided wireless artificial intelligence: embedding expert knowledge in deep neural networks for wireless system optimization," IEEE Vehicular Technology Magazine, vol. 14, no. 3, pp. 60-69, 2019.

[20] Y. Dai, D. Xu, S. Maharjan, G. Qiao, and Y. Zhang, “Artificial intelligence empowered edge computing and caching for internet of vehicles," IEEE Wireless Communications, vol. 26, no. 3, pp. 12-18, 2019.

[21] M. E. Morocho-Cayamcela, H. Lee, and W. Lim, "Machine learning for 5G/B5G mobile and wireless communications: potential, limitations, and future directions," IEEE Access, vol. 7, no. 99, pp. 137184-137206, 2019.

[22] L. Caviglione, M. Gaggero, J. F. Lalande, W. Mazurczyk, and M. Urbański, "Seeing the unseen: revealing mobile malware hidden communications via energy consumption and artificial intelligence," IEEE Transactions on Information Forensics and Security, vol. 11, no. 4, pp. 799-810, 2016.

[23] C. L. I, Q. Sun, Z. Liu, S. Zhang, and S. Han, "The big-datadriven intelligent wireless network: architecture, use cases, solutions, and future trends," IEEE Vehicular Technology Magazine, vol. 12, no. 4, pp. 20-29, 2017.

[24] P. Pace, G. Fortino, Y. Zhang, and A. Liotta, "Intelligence at the edge of complex networks: the case of cognitive transmission power control," IEEE Wireless Communications, vol. 26, no. 3, pp. 97-103, 2019.
[25] D. Xue, "Processing-based interactive installation art desig$\mathrm{n}$-an example of a potentially hazardous odour identification device for the vision-impaired," Journal of Physics Conference Series, vol. 2021, no. 1, pp. 012010-012013, 2021.

[26] M. C. Zeh, F. Cheung, S. Heijdens, and P. S. Wilson, "Silent room: a (non) sensory interactive art installation," Journal of the Acoustical Society of America, vol. 140, no. 4, pp. 32943294, 2016.

[27] Q. Hu, H. Wang, N. Di, H. Chen, and D. Huang, "Implementation and simulation analysis of an intelligent data fusion algorithm in wireless sensor network," Chinese Journal of Sensors and Actuators, vol. 31, no. 2, pp. 283-288, 2018.

[28] X. Liu and W. P. Jin, "The relationship between interactive installation art and traditional art," TECHART Journal of Arts and Imaging Science, vol. 6, no. 2, pp. 29-31, 2019.

[29] J. Liu, "Science popularization-oriented art design of interactive installation based on the protection of endangered marine life-the blue whale," Journal of Physics Conference Series, vol. 1827, no. 1, pp. 012116-012118, 2021. 